\title{
Oxidant regulated inter-subunit disulfide bond formation between ASIC1a subunits
}

\author{
Xiang-ming Zha a,b, Runping Wanga,b, Dan M. Collierc, Peter M. Snyderb,c, John A. Wemmie ${ }^{\mathrm{d}, \mathrm{e}}$, and Michael J. Welsh ${ }^{\mathrm{a}, \mathrm{b}, \mathrm{c}, \mathrm{1}}$ \\ aHoward Hughes Medical Institute and Departments of bInternal Medicine, 'Molecular Physiology and Biophysics, and dPsychiatry, Roy J. and Lucille A. \\ Carver College of Medicine, University of lowa, lowa City, IA 52242; and eVeterans Affairs Medical Center, lowa City, IA 52242
}

Contributed by Michael J. Welsh, December 31, 2008 (sent for review November 26, 2008)

The acid-sensing ion channel-1a (ASIC1a) is composed of 3 subunits and is activated by a decrease in extracellular $\mathrm{pH}$. It plays an important role in diseases associated with a reduced $\mathrm{pH}$ and production of oxidants. Previous work showed that oxidants reduce ASIC1a currents. However, the effects on channel structure and composition are unknown. We found that ASIC1a formed inter-subunit disulfide bonds and the oxidant $\mathrm{H}_{2} \mathrm{O}_{2}$ increased this link between subunits. Cys-495 in the ASIC1a C terminus was particularly important for inter-subunit disulfide bond formation, although other C-terminal cysteines contributed. Inter-subunit disulfide bonds also produced some ASIC1a complexes larger than trimers. Inter-subunit disulfide bond formation reduced the proportion of ASIC1a located on the cell surface and contributed to the $\mathrm{H}_{2} \mathrm{O}_{2}$-induced decrease in $\mathrm{H}^{+}$-gated current. These results indicate that channel function is controlled by disulfide bond formation between intracellular residues on distinct ASIC1a subunits. They also suggest a mechanism by which the redox state can dynamically regulate membrane protein activity by forming intracellular bridges.

ACCN2 | oxidation | trimer | acid-sensing ion channel

A cid-sensing ion channels (ASICs) are members of the degenerin/epithelial $\mathrm{Na}^{+}$channel family of non-voltagegated cation channels (1-3). There are 4 ASIC genes (ASIC1 to $A S I C 4$ ) and 2 splice variants (a and b) for $A S I C 1$ and $A S I C 2$. ASIC subunits have intracellular $\mathrm{N}$ and $\mathrm{C}$ termini, 2 transmembrane domains, and a large extracellular loop. A trimer of subunits comprises the channel (4), which can be composed of homologous or heterologous subunits. ASIC1a, -1b, $-2 \mathrm{a}$, and -3 are activated by extracellular protons and conduct $\mathrm{Na}^{+}$; ASIC1a homo-multimers also conduct $\mathrm{Ca}^{2+}(1-3)$.

ASIC1a is widely expressed in the brain (5-7). Within individual neurons, it localizes to the cell soma and to dendritic spines, where it mediates an acid-activated increase in $\left[\mathrm{Ca}^{2+}\right]_{\mathrm{i}}$ and regulates spine number $(8-10)$. ASIC1a is required for normal long-term potentiation (9), learning and memory (9), and both conditioned and innate fear-related behavior $(7,11$, 12). In addition to its role in normal brain physiology, ASIC1a contributes to several pathophysiological conditions. Disrupting the ASIC1a gene or inhibiting ASIC1a protected animals from ischemia-induced brain damage $(13,14)$, slowed disease progression in a mouse model of multiple sclerosis (15), and reduced disease in a mouse Parkinson model (16). ASIC1a also contributed to the termination of seizures (17). In all of these conditions, acidosis plays an important role $(15,17-19)$. In addition, these pathological conditions all generate free radicals, which in turn can contribute to the progression of disease (20-24).

Because acidotic and oxidizing environments coexist in diseases involving ASIC1a, several groups have tested the effect of redox reagents on ASIC1a function. Reducing agents increased ASIC1a current amplitude (25-27). Conversely, extracellular modification with a cysteine-oxidizing reagent 5,5'-dithiobis 2-nitrobenzoic acid (DTNB) decreased ASIC1a current $(26,27)$. At least part of the effect of DTNB depended on modifications within the first transmembrane domain. These studies suggested that oxidants can modulate ASIC1a function, and they emphasized the importance of cysteine modification in this cysteinerich protein. However, the underlying mechanisms are not well understood. ASIC1a has many conserved cysteines that could be targets for disulfide bond formation, yet it is unknown if oxidants affect those cysteines to alter ASIC1a structure. In addition, while previous studies have focused on modification of extracellular and transmembrane domains of ASIC1a, many oxidants are generated intracellularly, where they can also modify proteins (22). Given the signaling role of $\mathrm{H}_{2} \mathrm{O}_{2}$ and other oxidants in normal and abnormal brain function $(22,28)$ and the importance of ASIC1a in diseases associated with oxidative stress, we asked how $\mathrm{H}_{2} \mathrm{O}_{2}$ influences ASIC1a channel structure.

We discovered that oxidation enhances disulfide bond formation between ASIC1a subunits. We therefore asked if $\mathrm{H}_{2} \mathrm{O}_{2}$ regulated their formation, where the inter-subunit links occur, and how their formation affects the channel. Our findings of dynamic bridges between intracellular residues suggests an important mechanism for controlling protein activity in response to the redox state.

\section{Results}

ASIC1a Resides on the Cell Surface as a Trimer. Jasti et al. (4) crystallized chicken ASIC1 and found it to be a trimer. However, to obtain crystals, they truncated the subunit, removing most of the intracellular $\mathrm{N}$ and $\mathrm{C}$ termini. They also cross-linked the truncated ASIC1 subunits studied in solution and found a trimeric complex on Western blot analysis. We asked if a full-length ASIC1a channel located on the cell surface would have the same stoichiometry. We expressed ASIC1a in $\mathrm{CHO}$ cells, cross-linked using a membrane-impermeable cross-linker (sulfo-EGS), biotinylated surface proteins, precipitated with NeutrAvidin, and then blotted for ASIC1a. As the concentration of sulfo-EGS cross-linker increased, ASIC1a trimers became apparent on reducing gels (Fig. $1 A$ ). These data indicate that, when ASIC1a is on the cell surface, it exists as a trimer, consistent with the crystal structure of ASIC1 (4). $\mathrm{H}_{2} \mathrm{O}_{2}$ treatment did not affect this pattern, indicating that oxidation did not affect ASIC1a trimer formation (Fig. 1B).

ASIC1a Forms Inter-Subunit Disulfide Bonds. To learn whether inter-subunit disulfide bonds might contribute to the multimeric structure, we repeated the experiment, but lysed the cells in the presence of N-ethylmaleimide (NEM) to block reactive sulfhydryl groups and then analyzed using non-reducing conditions. Interestingly, these conditions revealed a band migrating be-

Author contributions: X.-m.Z., P.M.S., J.A.W., and M.J.W. designed research; X.-m.Z., R.W. D.M.C., and J.A.W. performed research; X.-m.Z., R.W., D.M.C., P.M.S., J.A.W., and M.J.W. analyzed data; and X.-m.Z. and M.J.W. wrote the paper.

The authors declare no conflict of interest.

Freely available online through the PNAS open access option.

${ }^{1}$ To whom correspondence should be addressed. E-mail: michael-welsh@uiowa.edu.

This article contains supporting information online at www.pnas.org/cgi/content/full/ 0813402106/DCSupplemental.

C 2009 by The National Academy of Sciences of the USA 
A

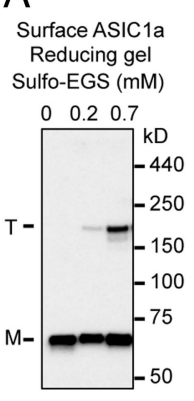

B

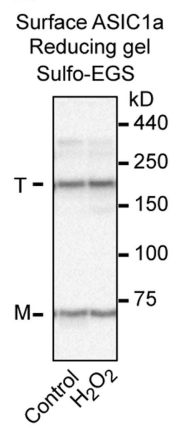

C Surface ASIC1a
Non-reducing gel Sulfo-EGS

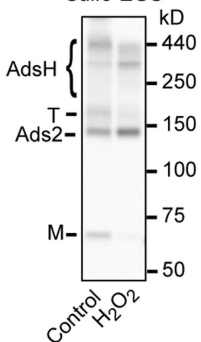

D
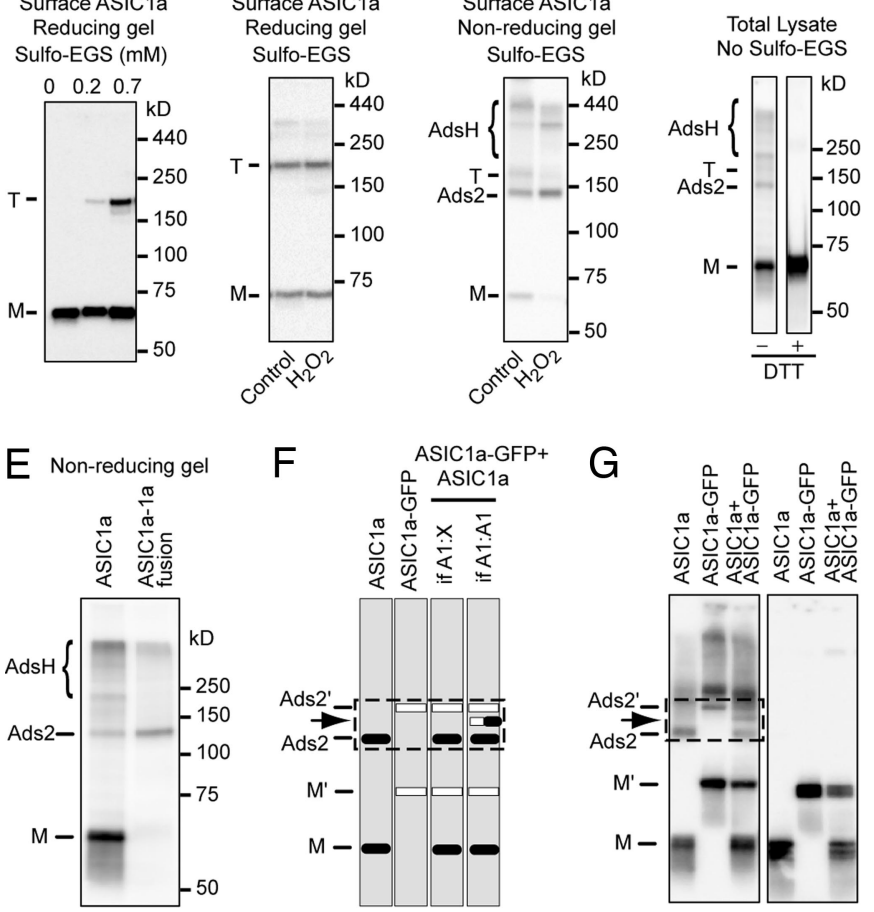

$\mathrm{F}$

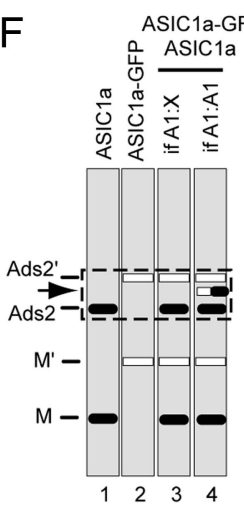

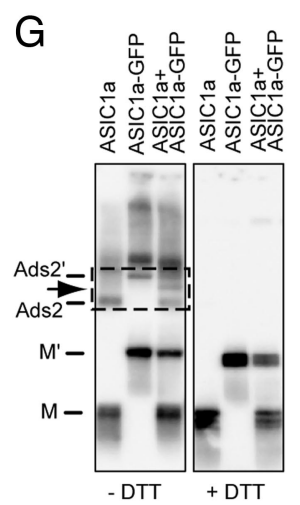

Fig. 1. ASIC1a forms inter-subunit disulfide bonds. (A) $\mathrm{CHO}$ cells were transfected with HA-ASIC1a, cross-linked with different concentrations of Sulfo-EGS as indicated, then biotinylated and lysed in the presence of 30 $\mathrm{mM}$ NEM to block all reactive sulfhydryl groups. Surface protein was pulled down with NeutrAvidin beads and resolved on reducing gel. Note that EGS is a membrane-impermeable cross-linker. ASIC1a monomeric bands are indicated by " $M$ " and trimers by " $T$." EGS dose-dependently revealed the presence of surface trimers. ( $B$ and $C$ ) $\mathrm{CHO}$ cells transfected with HA-ASIC1a were treated with $10 \mathrm{mM} \mathrm{H}_{2} \mathrm{O}_{2}$ at $4{ }^{\circ} \mathrm{C}$ for 10 min and cross-linked with 0.7 $\mathrm{mM}$ sulfo-EGS. Following surface protein precipitation, samples were treated with either reducing reagents $(B ; 200 \mathrm{mM}$ DTT or $10 \% \beta$-mercaptoethanol, both of which reduce disulfide bonds but do not affect the covalent bonds formed between sulfo-EGS and the lysine side chain) or non-reducing sample buffer $(C)$. Proteins were then separated on $7.5 \%$ or $10 \%$ SDS/PAGE. In all experiments, no cross-linking or reducing agent was present in the running buffer or PAGE gel. Note the appearance of multiple slowly migrating bands on a non-reducing gel. For convenience of description, we refer to the band between 150 and 100 kDa as "Ads2" for ASIC1a disulfide-linked subunit 2 and the bands above the trimer as "AdsH" for ASIC1a disulfide-linked subunit $\mathrm{H}$; see text. $(D)$ Western blot of total lysate of ASIC1a transfected $\mathrm{CHO}$ cells under non-reducing or reducing conditions. Reducing conditions decreased the relative amount of Ads2 from $11 \% \pm 5 \%$ to $0.2 \% \pm 0.1 \%$ and the relative amount of AdsH from $22 \% \pm$ $6 \%$ to $0.4 \% \pm 0.4 \%$. (E) Constructs encoding either an untagged ASIC1a or an ASIC1a-ASIC1a fusion protein were transfected into $\mathrm{CHO}$ cells. Cells were lysed in the presence of NEM and analyzed using non-reducing conditions. Note that Ads2 migrated at the same position as the ASIC1aASIC1a fusion protein. $(F)$ Illustration showing hypothetical migration of monomers $(M)$ and dimers (Ads2) when ASIC1a and GFP-tagged ASIC1a are expressed alone or together. For simplicity, we do not address more slowly migrating bands. Migration of GFP-tagged ASIC1a is indicated as $\mathrm{M}^{\prime}$ and Ads2' (lane 2). If Ads2 were a complex between ASIC1a and a different protein $X$, then only 2 bands-Ads 2 and Ads2'-would be seen when ASIC1 a and ASIC1a-GFP are co-expressed (lane 3). If Ads2 was a complex of ASIC1 a proteins, then an extra band between bands Ads2 and Ads2' would appear, corresponding to a complex of ASIC1a-s-s-ASIC1a-GFP (lane 4, arrow). (G) ASIC1a forms inter-subunit disulfide-linked subunits. Note the presence of an intermediate band between Ads2 and Ads2'. All slowly migrating bands were resistant to SDS but sensitive to DTT. We noticed that, on some gels, the monomeric band appeared as a doublet or triplet. We speculate that this may result from variations in modification or processing of ASIC1a.
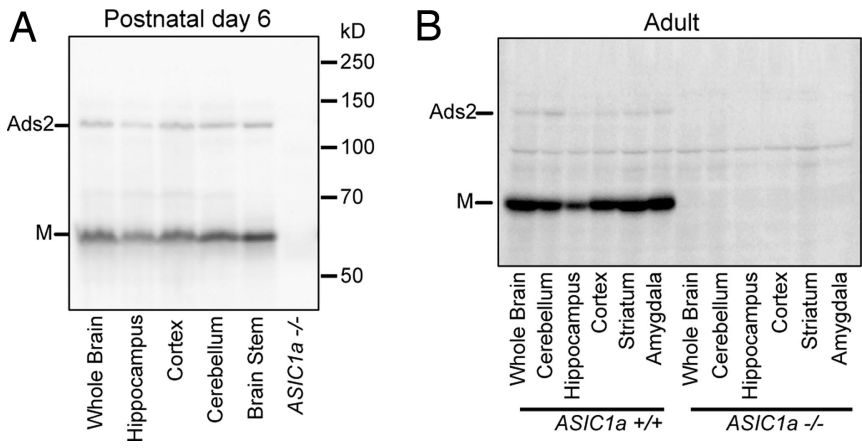

Fig. 2. ASIC1a forms disulfide-linked multimers in vivo. (A) Western blot of ASIC1a in WT and ASIC1a-I- brain from P6 mice. In addition to the presence of monomeric bands $(M)$, an Ads2 band was present in all brain regions examined. ASIC1a-null brain showed neither band. (B) Western blot of ASIC1a in adult mice. Note that the Ads 2 band is fainter compared with those from P6 mice $(A)$. Blot shown is over-exposed to better demonstrate the Ads2 band. Data are representative of $4 \mathrm{P} 6$ and 2 adult mice.

tween trimers and monomers and bands migrating more slowly than trimers (Fig. $1 C$ ). Treatment with $\mathrm{H}_{2} \mathrm{O}_{2}$ increased the fraction of ASIC1a in these bands, with a concomitant decrease of the monomer and trimer bands. These same bands appeared on non-reducing gels of total cell lysate in the absence of cross-linkers, and reducing conditions (i.e., DTT) eliminated them (Fig. 1D). Their sensitivity to reduction suggests that the bands other than the trimer and monomer represent ASIC1a subunits linked by disulfide bonds.

The most prominent band above the monomer migrated with a molecular mass expected for 2 ASIC1a subunits. In subsequent studies, we focused on this band to explore disulfide links between the subunits. For convenience in description, we refer to it as ASIC1a disulfide-linked subunit 2 (Ads2), and we refer to the species migrating more slowly than the trimer as ASIC1a disulfide-linked subunit $\mathrm{H}$ (AdsH).

The migration of Ads 2 suggested it contained 2 ASIC1a subunits. We did 2 sets of experiments to test this. We constructed a cDNA encoding an ASIC1a-ASIC1a fusion protein and found that the fusion protein migrated at the same position as the Ads2 band (Fig. $1 E$ ). To eliminate the possibility that Ads2 represented a complex between ASICla and another protein of similar molecular mass, we co-transfected cells with ASIC1a $(\approx 60 \mathrm{kDa})$ plus a GFP-tagged ASIC1a $(\approx 90 \mathrm{kDa})$. The boxed region of Fig. $1 F$ illustrates the potential outcomes. If Ads 2 is an oligomer between ASIC1a and a different protein "X," then we expected to see 2 bands at the Ads2 position: one for disulfide bonded ASIC1a-s-s-X and one for the ASIC1a-GFP-s-s-X (Fig. $1 F$, lane 3). Conversely, if ASIC1a forms inter-subunit disulfide bonds, then 3 bands should appear: one for ASIC1a-s-s-ASIC1a (Ads2), one for ASIC1a-GFP-s-s-ASIC1a-GFP (Ads2'), and one intermediate band for ASIC1a-s-s-ASIC1a-GFP (arrow, Fig. $1 F$, lane 4). For clarity, we do not show hypothetical products for AdsH. Fig. $1 G$ shows the results of this experiment. An intermediate band appeared when we co-expressed ASIC1a and GFP-tagged ASIC1a. These data indicate that Ads2 is an ASIC1a-s-s-ASIC1a complex linked by inter-subunit disulfide bonds, and further suggest that AdsH bands are higher-order ASIC1a oligomers (as discussed further later).

ASIC1a Forms Inter-Subunit Disulfide Bonds in Vivo. To learn if ASIC1a forms inter-subunit disulfide links in vivo, we blotted ASIC1a from various brain regions of postnatal day 6 (P6) and adult mice (Fig. $2 A$ and $B$ ). All brain regions examined showed both ASIC1a monomers and Ads2, but little AdsH. These data likely underestimate the frequency of inter-subunit 

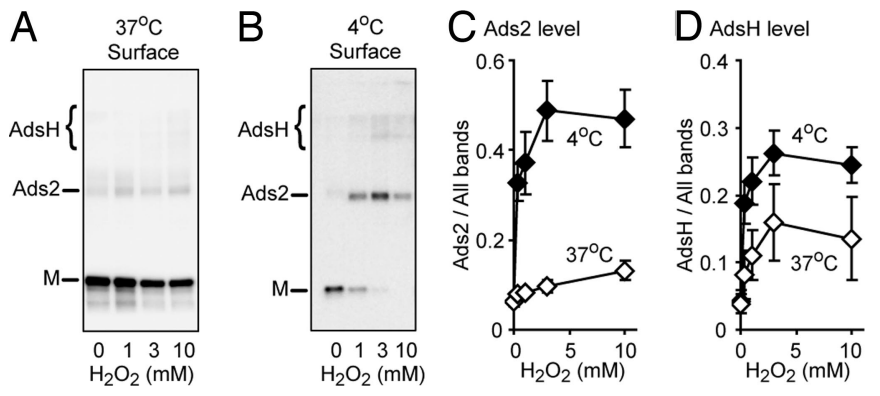

Fig. 3. Oxidation increases formation of inter-subunit disulfide bonds. ( $A$ and $B$ ) Effect of $\mathrm{H}_{2} \mathrm{O}_{2}$ on ASIC1a Ads2 formation at $37{ }^{\circ} \mathrm{C}(A)$ or $4{ }^{\circ} \mathrm{C}(B)$. ASIC1a-transfected cells were treated with different concentrations of $\mathrm{H}_{2} \mathrm{O}_{2}$ for $15 \mathrm{~min}$. After the indicated treatments, surface protein was biotinylated, isolated by NeutrAvidin pull-down, and analyzed under non-reducing conditions. (C) Quantification of ratio of Ads2 to total (estimated as monomer plus Ads2 plus AdsH). (D) Quantification of ratio of AdsH to total.

disulfide bonds because the ASIC1a antibody was directed against the ASIC1a C terminus and has reduced recognition of the disulfide-bonded form [supporting information (SI) Fig. S1].

Oxidation Regulates ASIC1a Inter-Subunit Disulfide Bond Formation and Surface Expression. To better understand how $\mathrm{H}_{2} \mathrm{O}_{2}$ affects inter-subunit disulfide bond formation, we measured the ratio of Ads2 to total ASIC1a on the cell surface. At $37^{\circ} \mathrm{C}, \mathrm{H}_{2} \mathrm{O}_{2}$ increased the proportion of ASIC1a in the Ads 2 and AdsH forms (Fig. $3 A, C$, and $D$ ). However, the relative increase was small, and we wondered if the effect was partially masked by differential trafficking of inter-subunit disulfide-linked and unlinked forms. To test this possibility, we repeated the experiment at $4{ }^{\circ} \mathrm{C}$ to eliminate channel trafficking. At $4{ }^{\circ} \mathrm{C}, \mathrm{H}_{2} \mathrm{O}_{2}$ markedly increased the proportion of Ads 2 and AdsH ASIC1a (Fig. $3 B$ and $C)$. In the absence of $\mathrm{H}_{2} \mathrm{O}_{2}$, approximately $10 \%$ of ASIC1a migrated in the Ads 2 plus Ads $\mathrm{H}$ forms, whereas with $1 \mathrm{mM}$ $\mathrm{H}_{2} \mathrm{O}_{2}$, approximately $59 \%$ of surface subunits were linked by inter-subunit disulfide bonds. These data indicate that disulfide linkage between ASIC1a subunits is a dynamic process promoted by $\mathrm{H}_{2} \mathrm{O}_{2}$. Importantly, this process occurs at the cell surface, which suggests a possible physiological mechanism for regulating ASIC activity.

These data also suggested that inter-subunit disulfide-linked forms may have reduced surface expression. We therefore predicted that oxidation would reduce the proportion of ASIC1a on the cell surface. To assess all cell surface ASIC1a (including Ads 2 and $\mathrm{AdsH}$ ) as accurately as possible, we treated surface and total fractions with $200 \mathrm{mM}$ DTT or $10 \% \beta$-mercaptoethanol, which reduced almost all Ads 2 and $\mathrm{AdsH}$ to a single monomeric band. At $37{ }^{\circ} \mathrm{C}$ but not $4{ }^{\circ} \mathrm{C}, 15$ min of treatment with $\mathrm{H}_{2} \mathrm{O}_{2}$ reduced the proportion of ASIC1a present on the cell surface in a concentration-dependent manner (Fig. $4 A$ and $B$ ).

C-Terminal Cysteines Form Inter-Subunit Disulfide Bonds. The ASIC1a crystal structure shows that the extracellular cysteines (except for Cys-70 and Cys-275) form intra-subunit disulfide bonds, and none of the extracellular cysteines in neighboring subunits sit close enough to form disulfide bonds between adjacent subunits (4). Consistent with this prediction, when we mutated each of the extracellular or transmembrane cysteines, none disrupted the formation of Ads2 or AdsH (not shown). However, ASIC1a also contains 4 highly conserved cysteines (Cys-464, Cys-469, Cys-495, and Cys-526) in its C terminus (Fig. $\mathrm{S} 2$ ), a region that was deleted in the crystal structure.

We hypothesized that one or more of these C-terminal cysteines contribute to oxidation-induced ASIC1a inter-subunit disulfide bond formation and surface expression. As an initial
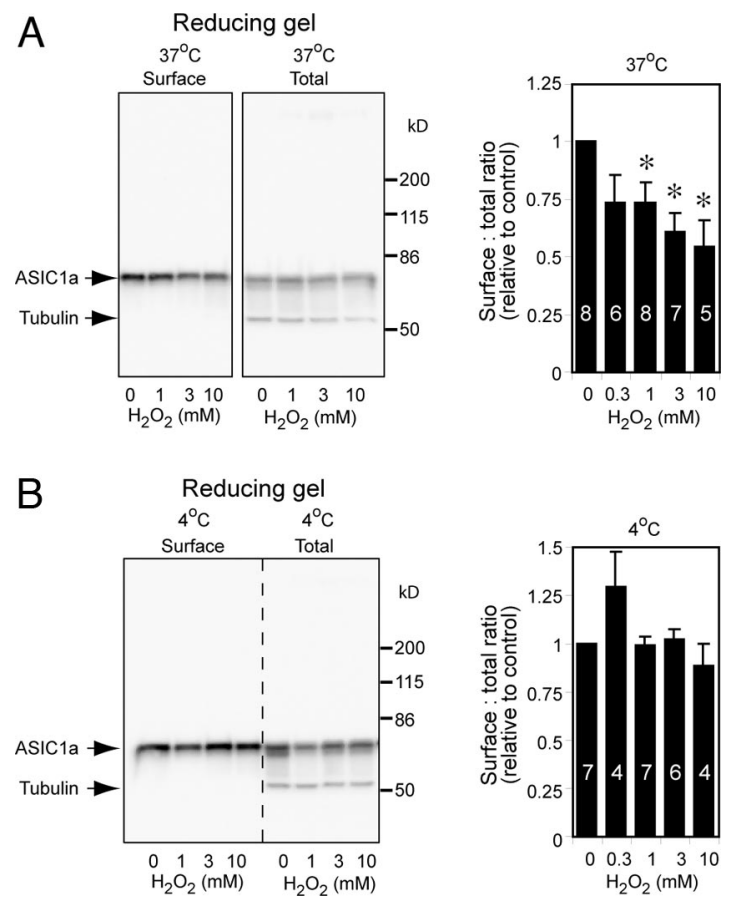

Fig. 4. $\mathrm{H}_{2} \mathrm{O}_{2}$ decreases surface expression of ASIC1a. ( $A$ and $B$ ) Representative Western blots showing surface and total ASIC1a in cells treated with indicated $\mathrm{H}_{2} \mathrm{O}_{2}$ concentrations for $15 \mathrm{~min}$ at $37^{\circ} \mathrm{C}(A)$ or $4{ }^{\circ} \mathrm{C}(B)$. To obtain more accurate quantification of ASIC1a levels, we boiled samples in the presence of $200 \mathrm{mM}$ DTT; after this treatment, ASIC1a runs predominantly as a monomeric band with little AdsH. We blotted the membrane with antiASIC1a antibody together with anti-tubulin antibody, which serves as a control for surface labeling. Surface levels of ASIC1a showed a concentration dependent decrease at $37{ }^{\circ} \mathrm{C}(A)$, but remain unchanged at $4{ }^{\circ} \mathrm{C}(B)$. Asterisks indicate differences from control $(P<0.05)$. Numbers on bars indicate the number of individual experiments.

test, we studied an ASIC1a mutant, ASIC1a-LLAA, which had the 4 C-terminal cysteines mutated to Leu, Leu, Ala, and Ala, respectively; we mutated Cys-464 and Cys-469 to Leu because the equivalent residues in ASIC2 are Leu. Eliminating the C-terminal cysteines abolished $\mathrm{H}_{2} \mathrm{O}_{2}$-induced Ads2 and AdsH formation at the cell surface (Fig. 5A). These data suggest that C-terminal cysteines are critical for forming inter-subunit disulfide bonds.

If inter-subunit disulfide bond formation is responsible for reducing the proportion of ASIC1a that resides on the cell surface, then we reasoned that mutating the $\mathrm{C}$-terminal cysteines would prevent this effect. We found that the LLAA mutation abolished the $\mathrm{H}_{2} \mathrm{O}_{2}$-induced reduction in the proportion of ASIC1a on the cell surface (Fig. 5B). These results suggest that the effect of oxidation on ASIC1a surface levels was caused by inter-subunit disulfide bond formation between C-terminal cysteines.

To identify which cysteines form inter-subunit disulfide bonds, we added them back one by one to ASIC1a-LLAA. The presence of Cys-495 restored $\mathrm{H}_{2} \mathrm{O}_{2}$-induced Ads 2 but not AdsH formation (Fig. $5 C$ ). None of the other 3 cysteines added back to ASIC1aLLAA restored the inter-subunit disulfide bonds. As an additional test, we mutated each C-terminal cysteine in ASIC1a individually. ASIC1a-C495A, but none of the other 3 mutants, reduced the proportion of ASIC1a migrating as Ads2 and AdsH following oxidation (Fig. 5D). These data indicate that Cys-495 is sufficient to generate the inter-subunit disulfide bonds in the Ads2 species, and they indicate that Cys-495 is necessary for normal, albeit not all, inter-subunit disulfide bond formation. 
A
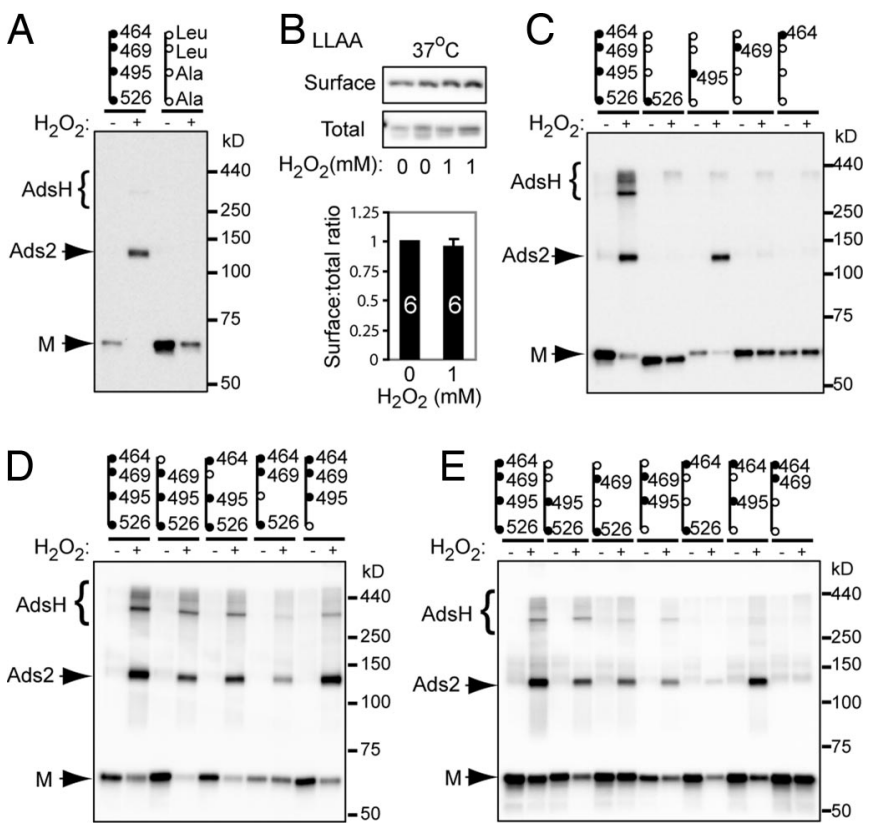

Fig. 5. C-terminal cysteines link ASIC1a subunits. (A) WT ASIC1a and cysteine mutants were treated with or without $10 \mathrm{mM} \mathrm{H}_{2} \mathrm{O}_{2}$ for 15 min at $4{ }^{\circ} \mathrm{C}$. Surface protein was analyzed under non-reducing conditions. The C-terminal tails are shown (Top), with closed circles indicating WT cysteines, open circles indicating mutated amino acid, and numbers indicating residue position. (B) Effect of $\mathrm{H}_{2} \mathrm{O}_{2}$ on surface expression of LLAA mutant. Cells were treated for 15 min with $\mathrm{H}_{2} \mathrm{O}_{2}$ and analyzed using reducing conditions. Numbers on bars indicate number of experiments. (C-E) Effect of $\mathrm{H}_{2} \mathrm{O}_{2}$ on formation of inter-subunit disulfide bonds in ASIC1a and variants with mutated C-terminal cysteines. Experimental conditions and mutations are as indicated in $B$. Data are from ASIC1a variants containing either a single intact Cys (C), a single Cys mutated and the other 3 residues intact $(D)$, or 2 of the Cys mutated $(E)$.

The failure of Cys-495 alone to restore AdsH (Fig. 5C) and the persistence of some Ads2 in ASIC1a-C495A (Fig. 5D) suggested that other cysteines might be involved in forming inter-subunit disulfide bonds. To test further this possibility, we studied the effect of double cysteine mutations in ASIC1a. When Cys-495 was present, adding either Cys-526, Cys-469, or Cys-464 increased AdsH formation (Fig. $5 E$ ). In addition, the combination of Cys-526 and Cys-469 partially restored the effect of $\mathrm{H}_{2} \mathrm{O}_{2}$ in the absence of Cys-495. These data show that, although Cys-495 is the most important residue for generating inter-subunit disulfide bonds, other C-terminal cysteines also participate or can compensate when Cys-495 is missing.

Oxidation May Link ASIC1a Trimers into Higher-Order Oligomers. Our data suggested that ASIC1a trimer formation and inter-subunit disulfide bond formation are distinct processes, a conclusion consistent with the ASIC1 crystal structure, which revealed no disulfide bonds between subunits missing the C termini (4). We further tested this hypothesis by asking if trimer formation was affected by the LLAA mutations, which prevent formation of inter-subunit disulfide bonds. We crosslinked ASIC1a with sulfo-EGS, biotinylated the cell surface, and precipitated with NeutrAvidin. Analysis under reducing conditions revealed the same pattern of trimers and monomers in both WT ASIC1a and ASIC1a-LLAA (Fig. 6A). These data further indicate that trimer formation is independent of oxidation and inter-subunit disulfide bond formation. Examination under non-reducing conditions showed that $\mathrm{H}_{2} \mathrm{O}_{2}$ increased the proportion of ASICla migrating as Ads2 at the expense of its migration as trimers (Fig. $1 C$ and Fig. $6 B$ ). The LLAA mutations abolished this effect (Fig. $6 B) . \mathrm{H}_{2} \mathrm{O}_{2}$ also increased the relative amount of AdsH of WT
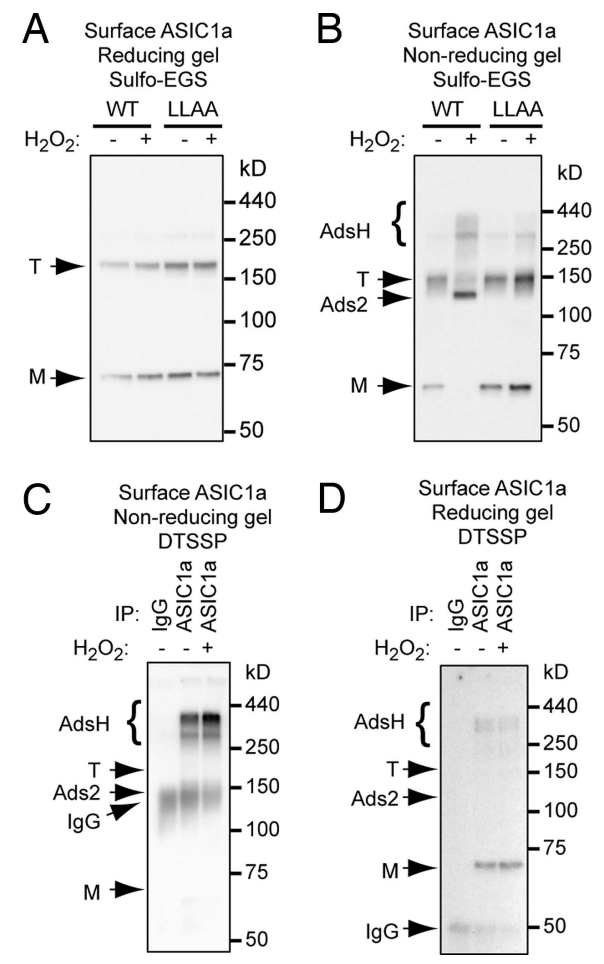

Fig. 6. Inter-subunit disulfide bonds may form higher-order ASIC1a complexes. WT ASIC1a and LLAA mutants were expressed in $\mathrm{CHO}$ cells and crosslinked with $0.7 \mathrm{mM}$ sulfo-EGS at $4{ }^{\circ} \mathrm{C}$ and then biotinylated. Surface fraction was analyzed by reducing $(A)$ or non-reducing $(B)$ Western blots. On a reducing gel, WT and LLAA mutant showed similar levels of trimer $(T)$ and monomer (M). On a non-reducing gel, $\mathrm{H}_{2} \mathrm{O}_{2}$ decreased band $\mathrm{T}$ and increased Ads 2 and Ads $\mathrm{H}$ of WT ASIC1a. In contrast, $\mathrm{H}_{2} \mathrm{O}_{2}$ had no effect on LLAA mutant. Blots are representative from 3 experiments. ( $C$ and $D$ ) Surface proteins were crosslinked with a membrane-impermeable reducible cross-linker, DTSSP. ASIC1a was immunoprecipitated with anti-HA antibody, followed by blotting for biotin. Under non-reducing conditions $(C)$, most of the protein ran at the band $\mathrm{H}$ position. Under reducing conditions $(D)$, most of the biotinylated protein migrated as the ASIC1a monomer. The position of IgG is also indicated.

ASIC1a but not ASIC1a-LLAA. These data suggest that oxidation links ASIC1a trimers into higher-order complexes.

We considered the possibility that the AdsH bands are a complex between ASIC1a and other surface proteins. To address this possibility, we biotinylated and cross-linked surface proteins with a membrane-impermeable, reducible cross-linker, DTSSP, and immunoprecipitated ASIC1a. Blotting for biotin under non-reducing conditions revealed most of the protein at the AdsH position (Fig. 6C). In contrast, under reducing conditions, most of the protein migrated as an ASIC1a monomer, although a small amount of AdsH persisted (Fig. 6D). If AdsH were a complex between ASIC1a and other surface proteins, then we might have expected to see additional prominent bands. These data, together with those in Fig. $6 B$, suggest that AdsH represents inter-trimer complexes. Nevertheless, we cannot entirely exclude the possibility that AdsH constitutes a complex of ASIC1a and other proteins.

Mutations That Prevent Inter-Subunit Disulfide Bond Formation Attenuate the Effect of $\mathbf{H}_{2} \mathbf{O}_{2}$ on ASIC1a Current. Previous studies showed that oxidation inhibited ASIC1a current, and much of the effect was attributed to modification at extracellular and transmembrane sites $(26,27)$. We found that $\mathrm{H}_{2} \mathrm{O}_{2}$ decreased ASIC1a current amplitude with a time course similar to that reported for the oxidizing compound DTNB (26) (Fig. $7 A$ and $C)$. However, our data predicted that a portion of the $\mathrm{H}_{2} \mathrm{O}_{2-}$ 
A ASIC1a

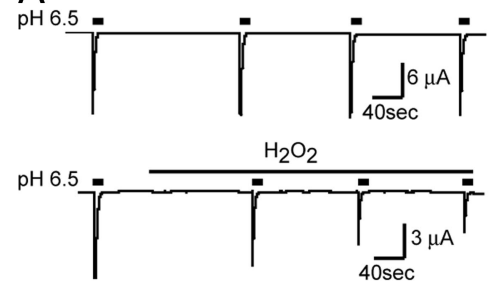

B ASIC1a-LLAA
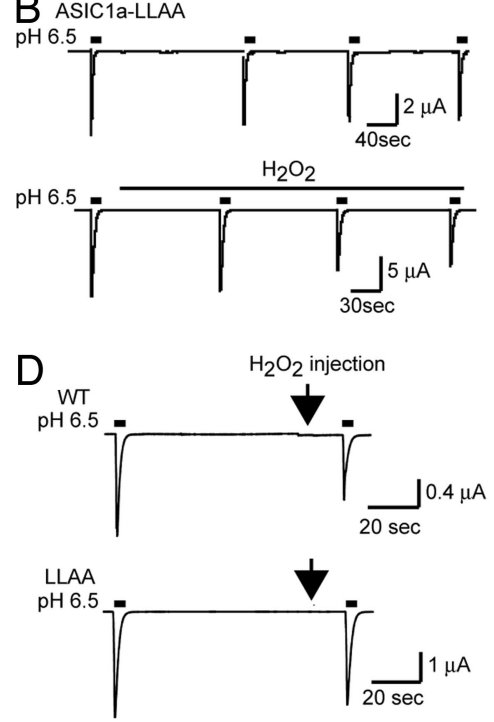

$\mathrm{E}$

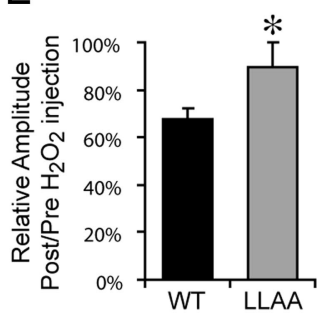

Fig. 7. Oxidation reduces ASIC1a current amplitude. ( $A$ and $B$ ) Representative traces from Xenopus oocytes injected with WT $(A)$ or LLAA $(B)$ ASIC1a and treated without (control) or with $20 \mathrm{mM} \mathrm{H}_{2} \mathrm{O}_{2}$ for 6 min. (C) Time course of current amplitude after $20 \mathrm{mM} \mathrm{H}_{2} \mathrm{O}_{2}$ addition $(n=16-22)$. Asterisks indicate $P<0.05$. (D) Representative traces showing effect of intracellular injection of $\approx 1.4 \mathrm{pl}$ of $\mathrm{H}_{2} \mathrm{O}_{2}$. $(E)$ Quantification of effect of intracellular $\mathrm{H}_{2} \mathrm{O}_{2}$ on current ( $n=8 \mathrm{WT}$ and $n=9$ LLAA experiments). Asterisk indicates $P=0.035$, unpaired $t$ test.

induced current reduction would result from inter-subunit disulfide bond formation and a reduced amount of ASIC1a on the cell surface. Indeed, $\mathrm{H}_{2} \mathrm{O}_{2}$ inhibited a smaller proportion of current in ASIC1a-LLAA channels (Fig. $7 B$ and $C$ ). To better assess the effect of intracellular oxidation, we injected $\mathrm{H}_{2} \mathrm{O}_{2}$ into oocytes and found that it reduced $\mathrm{H}^{+}$-gated current (Fig. $7 \mathrm{D}$ and $E)$. Although we cannot exclude the possibility that $\mathrm{H}_{2} \mathrm{O}_{2}$ might diffuse out and affect extracellular sites, the LLAA mutations minimized this effect (Fig. $7 D$ and $E$ ). These data suggest that $\mathrm{H}_{2} \mathrm{O}_{2}$ inhibited ASIC1a current in part by inducing inter-subunit disulfide bonds.

\section{Discussion}

Our data show that disulfide bonds between C-terminal cysteines link ASIC1a subunits. This was a surprising discovery because previous data showed that the $\mathrm{C}$ terminus was not required to form an ASIC1 structure (4), and we found that C-terminal cysteines were not needed to produce ASIC1a trimers on the cell surface or to generate functional channels. However, the Cterminal cysteines were a target for the oxidant $\mathrm{H}_{2} \mathrm{O}_{2}$; it induced inter-subunit links, reducing the amount of ASIC1a present on the cell surface and the $\mathrm{H}^{+}$-activated current.

Our data, combined with previous studies, indicate that $\mathrm{H}_{2} \mathrm{O}_{2}$ and other oxidants can regulate ASIC1a in at least 2 ways. Earlier work showed that oxidants decreased current amplitude, and the studies suggested that the modification was in the extracellular or transmembrane domains of ASIC1a $(26,27)$. We found a similar response to $\mathrm{H}_{2} \mathrm{O}_{2}$, an endogenous reactive oxygen species. Because $\mathrm{H}_{2} \mathrm{O}_{2}$ inhibited an ASIC1a variant that lacked intracellular cysteines, our data support the conclusion that oxidants have extracellular or transmembrane domain targets. However, our studies revealed a second target for $\mathrm{H}_{2} \mathrm{O}_{2}$ in the $\mathrm{C}$-terminal cysteines. Under basal conditions, we found some ASIC1a subunits linked to each other by disulfide bonds, and $\mathrm{H}_{2} \mathrm{O}_{2}$ markedly increased these interactions. Moreover, $\mathrm{H}_{2} \mathrm{O}_{2}$ decreased the amount of ASIC1a on the cell surface. Thus, $\mathrm{H}_{2} \mathrm{O}_{2}$ reduced ASIC1a current by 2 different mechanisms.

Previous studies have also demonstrated formation of cytosolic inter-subunit disulfide bonds (29). These include several neuronal ion channels. For example, skeletal muscle RyR-1 $\mathrm{Ca}^{2+}$-release channels form cytosolic inter-subunit disulfide links that increase channel activity (30). Other examples include shaker type $\mathrm{K}^{+}$channels (31) and cyclic nucleotide-gated (CNG) channels (32). One interesting aspect of the CNG channels is that ligand regulates formation of inter-subunit disulfide bonds; upon cGMP binding, $\mathrm{CNG}$ channels undergo a conformational change that brings intracellular $\mathrm{C}$-terminal cysteines from neighboring subunits close together to form disulfide bonds (32). All these studies demonstrate that inter-subunit disulfide bonds can form intracellularly between ion channel subunits.

Of the C-terminal cysteines, Cys-495 was the most important for generating disulfide bonds between ASIC1a subunits. To which of the 4 cysteines does Cys-495 link? If Cys-495-Cys-495 bonds were the only disulfide bridges, then several predictions would hold. Mutating Cys-495 would eliminate inter-subunit disulfide bonds. Mutating one or all of the other C-terminal cysteines would have no effect. In the absence of a cross-linker, a denaturing, non-reducing gel either with or without $\mathrm{H}_{2} \mathrm{O}_{2}$ treatment would show 2 linked subunits, not 3 or more. The studies in Fig. $5 D$ and $E$ and in Figs. $1 C, 3,5 B$, and 6 , respectively, are consistent with these predictions for Ads2, which represents 2 disulfide-bonded ASIC1a subunits. Although these results argue that Cys-495 has a critical role, the interpretation must certainly be more complex because the data indicate that other C-terminal cysteines are also involved in generating inter-subunit disulfide bonds. Because of the involvement of other cysteines, we cannot say whether the 2 disulfide-bonded ASIC1a subunits occur within a single trimeric channel, or whether the link occurs between subunits from separate trimers. Irrespective of the potential role of Cys-495 in generating links between trimers, the data suggest that the C-terminal cysteines also generate complexes (i.e., AdsH) that migrate more slowly than trimers. Thus, even though a single trimer likely forms a discrete ion channel, several channels may sit adjacent to each other in the membrane. Interestingly, clustering of neuronal ion channels such as the ionotropic glutamate receptor at postsynaptic membrane is thought to be important for its function $(33,34)$.

The crystal structure of ASIC1 demonstrated that the channel is comprised of 3 subunits (4). Our cross-linking data also revealed the presence of full-length ASIC1a trimers residing on the cell surface. However, previous studies of channel stoichiometry had suggested that degenerin/epithelial $\mathrm{Na}^{+}$channels were composed of 4 to 9 subunits (4). Our current data showing the existence of disulfide-linked higher-order complexes suggest a potential explanation for apparent discrepancies. They also suggest strategies for future studies designed to examine the consequences of higher-order structures and complexes of trimers.

There is substantial evidence that $\mathrm{H}_{2} \mathrm{O}_{2}$ and other oxidants play an important role in normal neuronal physiology. For example, endogenous $\mathrm{H}_{2} \mathrm{O}_{2}$ regulates neurotransmitter release in the striatum and substantial nigra (35) and GABAergic mini inhibitory post-synaptic currents in spinal cord neurons (36). $\mathrm{H}_{2} \mathrm{O}_{2}$ can also influence neuronal activity by modulating $\mathrm{Ca}^{2+}$ 
release from stores and subsequently regulating $\mathrm{Ca}^{2+}$-activated phosphatases, which in turn affect synaptic plasticity (28). Another reactive species, $\mathrm{NO}$, may also play an important role in normal neuronal function and can regulate ASIC function (37). Consistent with a role for disulfide-linked ASIC1a subunits, we also found them in brain, and the levels varied among brain regions. Thus, we speculate that disulfide bond formation between ASIC1a subunits may provide an important control of ASIC1a function.

$\mathrm{H}_{2} \mathrm{O}_{2}$ and other reactive oxygen species are also generated in several brain diseases that reduce $\mathrm{pH}$ and may involve ASIC1a, including stroke, multiple sclerosis, and Parkinson disease (1317). We speculate that oxidant-induced inter-subunit disulfide bond formation may reduce ASIC1a surface expression in these and related diseases. Reduced plasma membrane ASIC1a could attenuate acid-evoked increase in $\left[\mathrm{Ca}^{2+}\right]_{i}$, thereby providing a level of neuroprotection.

\section{Materials and Methods}

Detailed materials and methods are provided in the SI Text.

1. Kellenberger S, Schild L (2002) Epithelial sodium channel/degenerin family of ion channels: a variety of functions for a shared structure. Physiol Rev 82:735-767.

2. Waldmann R, et al. (1999) H(+)-gated cation channels. Ann N Y Acad Sci 868:67-76.

3. Wemmie JA, Price MP, Welsh MJ (2006) Acid-sensing ion channels: advances, questions and therapeutic opportunities. Trends Neurosci 29:578-586.

4. Jasti J, Furukawa H, Gonzales EB, Gouaux E (2007) Structure of acid-sensing ion channel 1 at 1.9 A resolution and low $\mathrm{pH}$. Nature 449:316-323.

5. Waldmann R, Champigny G, Bassilana F, Heurteaux C, Lazdunski M (1997) A protongated cation channel involved in acid-sensing. Nature 386:173-177.

6. Alvarez de la Rosa D, et al. (2003) Distribution, subcellular localization and ontogeny of ASIC1 in the mammalian central nervous system. J Physio/ 546:77-87.

7. Wemmie JA, et al. (2003) Acid-sensing ion channel 1 is localized in brain regions with high synaptic density and contributes to fear conditioning. J Neurosci 23:5496-5502.

8. Zha X-M, Wemmie JA, Green SH, Welsh MJ (2006) ASIC1a is a postsynaptic proton receptor that influences the density of dendritic spines. Proc Natl Acad Sci USA 103:16556-16561.

9. Wemmie JA, et al. (2002) The acid-activated ion channel ASIC contributes to synaptic plasticity, learning, and memory. Neuron 34:463-477.

10. Chai S, et al. (2007) A Kinase-anchoring Protein 150 and Calcineurin Are Involved in Regulation of Acid-sensing Ion Channels ASIC1a and ASIC2a.J Biol Chem 282:2266822677.

11. Wemmie J, Coryell M, Askwith C, Lamani E, Leonard S, Sigmund C, Welsh M (2004) Overexpression of acid-sensing ion channel $1 \mathrm{a}$ in transgenic mice increases fear-related behavior. Proc Natl Acad Sci USA 101:3621-3626.

12. Coryell M, et al. (2007) Targeting ASIC1a reduces innate fear and alters neuronal activity in the fear circuit. Biol Psychiatry 62:1140-1148.

13. Xiong ZG, et al. (2004) Neuroprotection in ischemia: blocking calcium-permeable acid-sensing ion channels. Cell 118:687-698.

14. Yermolaieva O, Leonard AS, Schnizler MK, Abboud FM, Welsh MJ (2004) Extracellular acidosis increases neuronal cell calcium by activating acid-sensing ion channel 1a. Proc Natl Acad Sci USA 101:6752-6757.

15. Friese MA, et al. (2007) Acid-sensing ion channel-1 contributes to axonal degeneration in autoimmune inflammation of the central nervous system. Nat Med 13:1483-1489.

16. Arias RL, et al. (2008) Amiloride is neuroprotective in an MPTP model of Parkinson's disease. Neurobiol Dis 31:334-341.

17. Ziemann AE, et al. (2008) Seizure termination by acidosis depends on ASIC1a. Nat Neurosci 11:816-822.

18. Siesjo BK, Katsura K, Kristian T Acidosis-related damage. Adv Neuro/ 1996;71:209-233.

19. Bowen BC, et al. (1995) Proton MR spectroscopy of the brain in 14 patients with Parkinson disease. AJNR Am J Neuroradiol 16:61-68.
Transfection, Biotinylation, and Cross-Linking. Transfection of $\mathrm{CHO}$ cells were done with Lipofectamine 2000. Surface biotinylation with NHS-biotin and cross-linking were performed at $4{ }^{\circ} \mathrm{C}$, following manufacturer's instruction (see SI Text).

Cell Lysis, Immunoprecipitation, and Western blot. Lysis of $\mathrm{CHO}$ cells or whole brain were performed in the presence of $30 \mathrm{mM}$ NEM. Standard immunoprecipitation and Western blot analysis were performed. Quantification was performed in ImageJ as described earlier (8) (see SI Text).

Electrophysiology. For electrophysiological studies, Xenopus oocytes were injected with ASIC1a cDNA and pH 6.5-activated currents were studied. Data are reported as mean \pm SEM (see SI Text)

ACKNOWLEDGMENTS. We thank Tami Nesselhauf and Pamela Hughes for cell culture in the in vitro models and Cell Culture Core, which was supported in part by National Heart, Lung and Blood Institute grant HL51670, the Cystic Fibrosis Foundation, and National Institutes of Diabetes and Digestive and Kidney Diseases grant DK54759). We thank Sarah Malerich, Yu F. Li, and Jill Haenfler for technical assistance. J.A.W. is supported in part by a Veterans Administration Research Center Development Award. X.M.Z. is a Research Specialist, R.W. is an Associate, and M.J.W. is an Investigator of the Howard Hughes Medical Institute.

20. Patel M (2004) Mitochondrial dysfunction and oxidative stress: cause and consequence of epileptic seizures. Free Radic Biol Med 37:1951-1962.

21. Emerit J, Edeas M, Bricaire F (2004) Neurodegenerative diseases and oxidative stress. Biomed Pharmacother 58:39-46.

22. Halliwell B (1992) Reactive oxygen species and the central nervous system. J Neurochem 59:1609-1623.

23. Warner DS, Sheng H, Batinic-Haberle I (2004) Oxidants, antioxidants and the ischemic brain. J Exp Biol 207:3221-3231.

24. Ghafourifar $\mathrm{P}$, et al. (2008) Mitochondria in multiple sclerosis. Front Biosci 13:3116-3126.

25. Cho JH, Askwith CC (2007) Potentiation of acid-sensing ion channels by sulfhydry compounds. Am J Physiol Cell Physiol 292:C2161-C2174.

26. Chu XP, Close N, Saugstad JA, Xiong ZG (2006) ASIC1a-specific modulation of acidsensing ion channels in mouse cortical neurons by redox reagents. J Neurosci 26:5329 5339.

27. Andrey F, Tsintsadze T, Volkova T, Lozovaya N, Krishtal O (2005) Acid sensing ionic channels: modulation by redox reagents. Biochim Biophys Acta 1745:1-6.

28. Kamsler A, Segal M (2004) Hydrogen peroxide as a diffusible signal molecule in synaptic plasticity. Mol Neurobiol 29:167-178.

29. Cumming RC, et al. (2004) Protein disulfide bond formation in the cytoplasm during oxidative stress. J Biol Chem 279:21749-21758.

30. Aracena-Parks $P$, et al. (2006) Identification of cysteines involved in S-nitrosylation S-glutathionylation, and oxidation to disulfides in ryanodine receptor type $1 . \mathrm{J} \mathrm{Biol}$ Chem 281:40354-40368.

31. Schulteis CT, Nagaya N, Papazian DM (1996) Intersubunit interaction between aminoand carboxyl-terminal cysteine residues in tetrameric shaker $\mathrm{K}+$ channels. Biochemis try 35:12133-12140

32. Rosenbaum T, Gordon SE (2002) Dissecting intersubunit contacts in cyclic nucleotidegated ion channels. Neuron 33:703-713.

33. Bolton MM, Blanpied TA, Ehlers MD (2000) Localization and stabilization of ionotropic glutamate receptors at synapses. Cell Mol Life Sci 57:1517-1525.

34. Kamboj S, Huganir RL (1998) Receptor clustering: activate to accumulate? Curr Bio 8:R719-R721.

35. Avshalumov MV, Bao L, Patel JC, Rice ME (2007) H2O2 signaling in the nigrostriatal dopamine pathway via ATP-sensitive potassium channels: issues and answers. Antioxid Redox Signal 9:219-231.

36. Takahashi A, Mikami M, Yang J (2007) Hydrogen peroxide increases GABAergic mIPSC through presynaptic release of calcium from IP3 receptor-sensitive stores in spinal cord substantia gelatinosa neurons. Eur J Neurosci 25:705-716.

37. Cadiou $\mathrm{H}$, et al. (2007) Modulation of acid-sensing ion channel activity by nitric oxide. Jeurosci 27:13251-13260. 